\title{
On the Motion Uniform in a Direction
}

\author{
by \\ T. Sato \\ Meteorological Research Institute \\ (Received June 12, 1951)
}

\begin{abstract}
Under four assumptions we integrated the equations of motion uniform in a direction, and obtained integrals in Cartesian coordinates, in the $x$-direction of which the motion is considered to be uniform. As in this case it is seen from the equation of continuity that momentum can be expressed by applying streamsurface functions, therefore we can get two integrals : one concerning momentum and the other concerning vorticity. The integral concerning energy can not be obtained, unless we assume the pressure distribution to be stationary.

If we further assume that the motion is stationary, then we get the same integrals as those which we derived before.
\end{abstract}

In discussing the motion, we adopt the following four assumptions;
(A) fluid is frictionless,
(B) fluid is autobarotropic,
(C) the effect of deflecting force is negligible,
(D) the state of motion is uniform in a direction.

The equation of continuity is

$$
\frac{\partial q}{\partial t}+\frac{\partial}{\partial y}(q v)+\frac{\partial}{\partial z}(q w)=0
$$

If we consider now a vector $(q, q v, q w)$ in the $(t, y, z)$ space, the above equations hows that the divergence of this vector is always zero in the space. Hence, applying stream-surface functions $\alpha(t, y, z)$ and $\beta(t, y, z)$, the vector can be expressed as

$$
q v=\nabla \alpha \times \nabla \beta,
$$

where $\nabla$ is with respect to $t, y$ and $z$, or in components

$$
\begin{aligned}
q & =\frac{\partial \alpha}{\partial y} \frac{\partial \beta}{\partial z}-\frac{\partial \alpha}{\partial z} \frac{\partial \beta}{\partial y} \equiv \frac{\partial(\alpha, \beta)}{\partial(y, z)}, \\
q v & =\frac{\partial \alpha}{\partial z} \frac{\partial \beta}{\partial t}-\frac{\partial \alpha}{\partial t} \frac{\partial \beta}{\partial z} \equiv \begin{array}{l}
\partial(\alpha, \beta) \\
\partial(z, t)
\end{array}, \\
q w & =\frac{\partial \alpha}{\partial t} \frac{\partial \beta}{\partial y}-\frac{\partial \alpha}{\partial y} \frac{\partial \beta}{\partial t} \equiv \frac{\partial(\alpha, \beta)}{\partial(t, y)} .
\end{aligned}
$$


Now we consider the geometrical meaning of these equations.

It is more comprehensible for geometrical interpretation to adopt the ordinary three-dimensional space $(x, y, z)$ than the space $(t, y, z)$. If we adopt the assumption: $\left(D^{\prime}\right)$ motion is stationary, that is, $\partial / \partial t=0$, instead of : (D) the state of motion is uniform in a direction, that is, $\partial / \partial x=0$, then the equation of continuity becomes instead of (1),

$$
\frac{\partial}{\partial x}(q u)+\frac{\partial}{\partial y}(q v)+\frac{\partial}{\partial z}(q w)=0 .
$$

As this equation shows that the vector ${ }_{\alpha} v$ representing momentum is non-divergent or solenoidal, it can be expressed by the solenoids $(\alpha, \beta)$ of two mutually independent scalar quantities $\alpha$ and $\beta$. The two surfaces

$$
\alpha(x, y, z)=\text { const }, \quad \beta(x, y, z)=\text { const }
$$

form two systems of stream-surfaces or a sysiem of stream-tubes. The direction of the vector $q v$ is expressed by the line of intersection of the two stream-surfaces and the magnitude is expressed by

$$
\left|{ }_{q} \boldsymbol{v}=\right| \boldsymbol{\nabla} \alpha \times \nabla \beta|=| \frac{d \alpha}{d n_{a}}|| \frac{d \beta}{d n_{\beta}}|| \sin \varepsilon \mid,
$$

where $\varepsilon$ is the angle of intersection of the two stream-surfaces

$$
\alpha=\text { const and } \beta=\text { const. }
$$

In our problem $\alpha$ and $\beta$ are stream-surface functions in the space $(t, y, z)$. The state of motion at every time point is determined by a system of intersection of the surface $a=$ const. , that is, the $y z$-plane, with the systems of stream-surfaces (streamtubes). If we know the systems of these lines at $t=t_{0}, t_{1}, t_{2}, \cdots \cdots$, then the projection of motion on the $y z$-plane is fully determined. As we are now treating of the motion uniform in the $x$-direction, the three dimensional motion in the space $(x, y, z)$ at $t=t_{0}, t_{1}, t_{2}, \cdots \cdots$, is fully determined from the projection on the $y z$-plane.

If we further assume the motion to be stationary, then, putting $\beta=t$ in (2b). and (2c), and writing $\phi$ instead of $\alpha$, we get

$$
q v=\frac{\partial \psi}{\partial z}, \quad q w=-\frac{\partial \psi}{\partial y},
$$

in which $\phi$ expresses the stream-line function of the two-dimensional vector $(q v, q w)$. The state of motion in this case was fully discussed in our previous papers. As this is the case when the $\beta$-plane coincided with the $y z$-plane, therefore it appears on the $y z$-plane only the lines of intersection of the surfaces $\alpha=$ const. with the $y z$-plane. These lines of intersection become the stream-lines in this case.

Now we transform the equations of motion by applying the stream-surface functions $\alpha$ and $\beta$. The equations of motion are written under the initial assumptions (A), (B), (C) and (D), as follows

$$
\frac{\partial \boldsymbol{v}}{\partial t}+(\boldsymbol{v} \cdot \nabla) \boldsymbol{v}+\nabla \phi+s \nabla p=0,
$$

or in components 
(5a)

$$
\begin{aligned}
& \frac{\partial u}{\partial t}+v \frac{\partial u}{\partial y}+w \frac{\partial u}{\partial z}=0, \\
& \frac{\partial v}{\partial t}+v \frac{\partial v}{\partial y}+w \frac{\partial v}{\partial z}+s \frac{\partial p}{\partial y}=0, \\
& \frac{\partial w}{\partial t}+v \frac{\partial w}{\partial y}+w \frac{\partial w}{\partial z}+g+s \frac{\partial p}{\partial z}=0 .
\end{aligned}
$$

In order to derive integrals, we apply the following relation. The necessary and sufficient condition for the three variables $f, \alpha$ and $\beta$ with respect to $t, y$ and $z$ to be mutually connected by a functional relation, is that their Jacobian determinant is zero, that is

$$
\frac{\partial(f, \alpha, \beta)}{\partial(t, y, z)} \equiv\left|\begin{array}{lll}
\frac{\partial f}{\partial t} & \frac{\partial f}{\partial y} & \frac{\partial f}{\partial z} \\
\frac{\partial \alpha}{\partial} & \frac{\partial \alpha}{\partial t} & \frac{\partial \alpha}{\partial z} \\
\frac{\partial \beta}{\partial t} & \frac{\partial \beta}{\partial y} & \frac{\partial \beta}{\partial z}
\end{array}\right|=0 .
$$

In the case when the above relation (6) holds, the function $f$ can be expressed by using $\alpha$ and $\beta$ as follows

$$
f=F(\alpha, \beta),
$$

where $F(\alpha, \beta)$ is an arbitrary function of $\alpha$ and $\beta$ only.

The equation (6) as expressed

$$
\left\{\frac{\partial(\alpha, \beta)}{\partial(y, z)}\right\} \frac{\partial t}{\partial t}+\left\{\frac{\partial(\alpha, \beta)}{\partial(z, t)}\right\} \frac{\partial f}{\partial y}+\left\{\frac{\partial(\alpha, \beta)}{\partial(t, y)}\right\} \frac{\partial f}{\partial z}=0
$$

can be transformed in our case, applying (2), into

$$
\frac{\partial f}{\partial t}+v \frac{\partial f}{\partial y}+w \frac{\partial f}{\partial z} \equiv \frac{d f}{d t}=0 \text {. }
$$

Thus if the function $f$ satisfies (8), then it can be expressed by (7). This means physically that a conservative quantity if can be expressed as a function of streamsurface functions only.

We integrate the equations (5) of motion by applying these relations،

First, (5a) is transformed as follows

$$
\frac{\partial u}{\partial t}+v \frac{\partial u}{\partial y}+w \frac{\partial u}{\partial z} \equiv \frac{d u}{d t}=0,
$$

hence, we get from (8)

$$
\text { (9) } u=V(\alpha, \beta) \text {, }
$$

where $V(\alpha, \beta)$ is an arbitrary function of $\alpha$ and $\beta$ only. This expresses an integral with respect to momentum (the $x$-component of.).

If we put $\beta=t(=$ const $)$ in this equation and write $\psi$ instead of $\alpha$, then we get

$$
u=V(\psi) \text {, }
$$

which expresses the law of conservation of momentum in the case of stationary motion, and coincides with that which we derived before.

Next, we calculate $\frac{\partial}{\partial z}(5 b)-\frac{\partial}{\partial y}(5 c)$, then we get 


$$
\left(\frac{\partial}{\partial t}+v \frac{\dot{\partial}}{\partial y}+w \frac{\partial}{\partial z}+\frac{\partial v}{\partial y}+\frac{\partial w}{\partial z}\right)\left(\frac{\partial w}{\partial y}-\frac{\partial v}{\partial z}\right)=0 .
$$

On the other hand

$$
\left(\frac{\partial}{\partial t}+v \frac{\partial}{\partial y}+w \frac{\partial}{\partial z}\right)\left\{s\left(\frac{\partial w}{\partial y}-\frac{\partial v}{\partial z}\right)\right\}=s\left(\frac{\partial}{\partial t}+v \frac{\partial}{\partial y}+w \frac{\partial}{\partial z}+\frac{\partial v}{\partial y}+\frac{\partial w}{\partial z}\right)\left(\frac{\partial w}{\partial y}-\frac{\partial v}{\partial z}\right),
$$

where $s=\frac{1}{q}$. Thus we can derive the following integral

$$
-s\left(\frac{\partial w}{\partial y}-\frac{\partial v}{\partial z}\right)=\Psi(\alpha, \beta)
$$

where $\Psi(\alpha, \beta)$ expresses an arbitrary function of $\alpha$ and $\beta$ only. This equation is written also in $\alpha$ and $\beta$ as follows

$$
\begin{aligned}
s\left[s \frac{\partial}{\partial y}\left\{\frac{\partial(\alpha, \beta)}{\partial(y, t)}\right\}+s \frac{\partial}{\partial z}\left\{\frac{\partial(\alpha, \beta)}{\partial(z, t)}\right\}\right. & +\frac{\partial s}{\partial y} \frac{\partial(\alpha, \beta)}{\partial(y, t)} \\
& \left.+\frac{\partial s}{\partial z} \frac{\partial(\alpha, \beta)}{\partial(z, t)}\right]=\Psi(\alpha, \beta) .
\end{aligned}
$$

This is an equation with respect to vorticity.

Putting $\beta=t(=$ const $)$ and writing $\psi$ instead of $\alpha$, we get

$$
s\left(s \frac{\partial^{2} \psi}{\partial y^{2}}+s \frac{\partial^{2} \psi}{\partial z^{2}}+\frac{\partial s}{\partial y} \frac{\partial \psi}{\partial y}+\frac{\partial s}{\partial z} \frac{\partial \psi}{\partial z}\right)=\Psi(\psi),
$$

which expresses the law of conservation of vorticity (the $x$-component of) in the case of stationary motion, and coincides with that which we derived before.

Lastly we derive a relation concerning energy. Multiplying (5) with vector $v$ scalarly, we get, applying the assumption (B)

$$
\frac{d}{d t}\left(\frac{1}{2} \boldsymbol{v}^{2}\right)+\boldsymbol{v} \cdot \nabla\left(\phi+\int s d p\right)=0
$$

which is also transformed as follows

$$
\left(\frac{\partial}{\partial t}+v \frac{\partial}{\partial y}+w \frac{\partial}{\partial z}\right)\left(\frac{1}{2} v^{2}+\phi+\int s d p\right)=s \frac{\partial p}{\partial t},
$$

which can not be integrated in our way, unless we assume the distribution of pressure to be stationary.

If the field of pressure is stationary, then we get

$$
\frac{1}{2} v^{2}+\phi+\int s d p=E(\alpha, \beta),
$$

which can be rewritten as

$$
\frac{1}{2}\left[\left\{s \frac{\partial(\alpha, \beta)}{\partial(y, t)}\right\}^{2}+\left\{s \frac{\partial(\alpha, \beta)}{\partial(z, t)}\right\}^{2}+V^{2}\right]+g z+\int s d p=E(\alpha, \beta) .
$$

Putting $\beta=t(=$ const $)$ in this equation and writing $\psi$ instead of $\alpha$, we get

$$
\frac{1}{2}\left\{s^{2}\left(\frac{\partial \dot{\psi}}{\partial y}\right)^{2}+s^{2}\left(\frac{\partial \psi}{\partial z}\right)^{2}+V^{2}\right\}+g z+\int s d p=E(\psi),
$$

which expresses the law of conservation of energy in the case of stationary motion, and coincides with that which we derived before.

\section{Reference}

S 